\title{
Rethinking vaccine development as integral part of preparedness in the European Health Union
}

\author{
Marco Rizzi*
}

\section{Introduction}

In a 2004 editorial, The Lancet warned health authorities globally that public health preparedness requires "strong national public-health capacities [...] if countries are to maintain their sovereignty in public-health emergency situations." ${ }^{1}$ The editorial concluded by emphasising the imperative for public authorities to ensure "that incentives for vaccine production capacity are promoted alongside the revised IHR [International Health Regulations] and not side-lined in separate influenza preparedness programmes." ${ }^{2}$ Significantly, the editorial focused on the risk of an influenza pandemic, highlighting how surveillance and security measures would be inadequate against a "disease in which the period of infectivity precedes the onset of symptoms." ${ }^{3}$ As is now painfully obvious, this reasoning cannot be limited to influenza diseases.

This piece argues that the role of vaccines as essential tools for public health preparedness in the face of pandemic and epidemic diseases (PEDs) remains unachieved as the tally of COVID-19 confirmed cases and deaths grows by the day. ${ }^{4} \mathrm{It}$ is clear that a vaccine is critical for a return to safe and free flowing social interactions. The unprecedented amount of resources thrown at research on the SARS-CoV-2 virus prompts optimistic forecasts that a vaccine may be available in $2021 .{ }^{5}$ This would be an unprecedented timeframe for the development of a new vaccine, which normally takes between five and fifteen years. ${ }^{6}$ But is the current regulatory framework able to cater for the need to develop vaccines 'at pandemic speed'?

There is room to question the fitness for purpose of the current international regime of public health preparedness, governed by the WHO International Health Regulations 2005 (IHR), and its European alter ego, the EU Health Threats Decision 1082/2013/EU ${ }^{8}$. If public health preparedness can be metaphorically described as a 'tool box', the immunization 'tool' is currently given insufficient consideration. While immunization campaigns and vaccine $R \& D$ could reasonably be expected to

\footnotetext{
${ }^{*}$ Senior Lecturer, University of Western Australia Law School. I wish to thank the anonymous reviewers for their helpful comments, and Gatwiri M'mbijiwe for her excellent research assistance. All mistakes remain my own.

${ }^{1}$ The Lancet, 'Public-Health Preparedness Requires More than Surveillance' (2004) 364 The Lancet 1639.

2 Ibid.

${ }^{3}$ Ibid.

${ }^{4}$ Updated figures are retrievable from: Johns Hopkins University of Medicine, 'COVID-19 Dashboard by the Centre for Systems Science and Engineering at John Hopkins University of Medicine' (Coronavirus Resource Center) <https://coronavirus.jhu.edu/map.html> accessed 20 July 2020.

5 Tung Thanh Le and others, 'The COVID-19 vaccine development landscape' (2020) 19 Nature Reviews Drug Discovery 305-306 <https://www.nature.com/articles/d41573-020-00073-5> accessed 2 October 2020. An experimental vaccine from Oxford is showing promising signs, see Pedro M Folegatti and others, 'Safety and immunogenicity of the ChAd0x1 nCoV-19 vaccine against SARS-CoV-2: a preliminary report of phase $1 / 2$, single-blind, randomised controlled trial' (2020) The Lancet <https://doi.org/10.1016/S0140-6736(20)31604-4> accessed 2 October 2020.

${ }^{6}$ International Federation of Pharmaceutical Manufacturers and Associations, 'The Complex Journey of a Vaccine The Steps Behind Developing a New Vaccine' (IFPMA, July 2019) <https://www.ifpma.org/wpcontent/uploads/2019/07/IFPMA-ComplexJourney-2019_FINAL.pdf> accessed 2 October 2020.

${ }^{7}$ Nicole Lurie and others, 'Developing Covid-19 Vaccines at Pandemic Speed', New England Journal of Medicine (2020) 382(21) New England Journal of Medicine 1969.

${ }^{8}$ Decision No 1082/2013/EU of the European Parliament and of the Council on serious cross-border threats to health and repealing Decision No 2119/98/EC (Health Threats Decision) [2013] OJ L 293.
} 
feature prominently in any policy and/or strategic document addressing emerging health threats, vaccination occupies a subsidiary role, with a prevalent focus on risk management mechanisms.

Traditionally, the market-oriented and efficiency-based logic underpinning vaccine R\&D led by a strong private sector has taken precedence over public health protection and preparedness in this particular field. ${ }^{9}$ The trend has been challenged in recent years by a recognised increase in the incidence of both viral and bacterial infectious diseases. ${ }^{10}$ The EU's plans to move towards a European Health Union require a paradigm shift in the regulation of vaccine R\&D for emerging PEDs, which calls for greater public intervention and the introduction of dedicated regulatory pathways inspired by cooperation over competition.

\section{The fiddly concept of preparedness in international and EU public health, and the place of vaccines}

Preparedness is the key conceptual pillar developed in the context of global public health for the purpose of addressing the risk of pandemic or epidemic diseases (PEDs). It is impossible to provide a full account of the genesis and evolution of the concept and its deployment in public health, but it is worth making some brief observations. The idea of preparedness stems from an appreciation of the inherent limits of prevention in tackling a wide range of potential threats, including public health ones. ${ }^{11}$ This entails a conceptual shift from the objective of avoiding the occurrence of a future harmful event, to accepting its inevitability and being equipped to manage its unfolding and mitigate its consequences. ${ }^{12}$ Natural disasters are a good example of events that cannot be prevented, but that we can attempt to be prepared for. ${ }^{13}$ Preparedness in public health has been developed to harness the yearning of modern societies to regulate developing emergencies even in situations of high uncertainty, with in-built mechanisms for ongoing assessment and reassessment of vulnerabilities. ${ }^{14}$

The prevalence of preparedness dichotomy in public health can be observed both at the international and European levels in key instruments, specifically the IHR 2005 and the EU Health Threats Decision. The instruments laid out in Articles 5 to 14 of the IHR 2005 paint a telling picture of how public health preparedness has been understood over the past fifteen years. IHR preparedness provisions rely on an eminently reactive framework, attracting strong criticism for a number of reasons spanning the necessary reliance on individual countries' resources to implement

${ }^{9}$ The key issue is that the main drivers of R\&D investment are the probability of success of a new vaccine, coupled with the potential extent of demand. See Andrew Lo, Kien Wei Siah and Chi Heem Wong, 'Estimating Probabilities of Success of Vaccine and Other Anti-Infective Therapeutic Development Programs' (2020) 1 Harvard Data Science Review <https://doi.org/10.1162/99608f92.e0c150e8> accessed 2 October 2020. On the impact of estimated demand, see the discussion below regarding the R\&D process for the Ebola, SARS and MERS vaccines.

${ }^{10}$ As highlighted in numerous recent industry reports, see for example: Summant Ugalmugle and Rupali Swain, 'Global Vaccines Market Size By Age Group (Pediatric, Adult), By Technology (Conjugate, Attenuated, Inactivated, Recombinant, Toxoid), By Disease (Cancer, Hepatitis, Pneumococcal Disease, DTP, Dengue, Influenza, Human Papilloma Virus, Meningococcal Disease, Polio, Rotavirus), Regional Outlook, Application Potential, Price Trends, Competitive Market Share \& Forecast, 2019 - 2025' (2019) Global Market Insights <https://www.gminsights.com/industry-analysis/vaccines-market> accessed 2 October 2020.

${ }^{11}$ Andrew Lakoff, 'The Generic Biothreat, or, How we Became Unprepared' (2008) 23(3) Cultural Anthropology 399; Andrew Lakoff, 'Preparing for the Next Emergency' (2007) 19 Public Culture 247.

12 Lakoff, 'Preparing for the Next Emergency' (n 8) 253; Peter Adey and Ben Anderson, 'Anticipating Emergencies: Technologies of Preparedness and the Matter of Security' (2012) 43 Security Dialogue 99.

${ }^{13}$ For a thorough discussion of preparedness see Andrew Lakoff, Unprepared - Global Health in a Time of Emergency (UCP 2017).

${ }^{14}$ Mark Flear, "'Technologies of Reflexivity": Generating Biopolitics and Institutional Risk to Supplement Global Public Health Security' (2017) 8 European Journal of Risk Regulation 658, 665. 
the required detection and communication operations, as well as their good faith. ${ }^{15} \mathrm{~A}$ different type of criticism focuses on the inherent bias of the IHR 2005, which were arguably premised on the assumption that infectious diseases would come from the 'Global South', creating a need to protect the 'Global North'. ${ }^{16}$ The European Health Threats Decision goes in a similar direction, emphasising early-warning systems, communications, risk assessment and management, and procurement of medical countermeasures. ${ }^{17}$

The focus on preparedness in global and European public health policy efforts to confront emerging health threats can also be tied to a parallel theme: the increasing link between health and security policy. ${ }^{18}$ Biosecurity, or the securitisation of public health, can be traced back to the mid 1990s, responding to factors such as the fear of bioweapons and terrorism, the continued global spread of HIV/AIDS and the growing awareness of the risk of globalised spread of emerging diseases (accompanied by the uneasy realisation that developed and rich countries were no longer immune). In this context, securitisation functioned as a way to elevate the saliency of global public health issues. ${ }^{19}$ In the EU context, the rise of the health-security nexus was driven by concurrent factors including a tendency of EU policy-making to be events-driven and reactive, a measure of EU entrepreneurship, and a generalised shift towards securitisation in a number of policy areas. ${ }^{20}$ The Health Threats Decision, with its specific focus on coordination, cooperation and centralisation of response mechanisms, was arguably developed with the specific intent of bridging the fields of public health and security. ${ }^{21}$ Interestingly, this seems to have resulted in a peculiar hiatus between a securitised public health preparedness framework, and the regulatory mechanisms overseeing research, development and approval of therapeutic countermeasures including immunization.

Immunization through vaccines is the primary method of infectious disease prevention. It is the most cost-effective way of reducing the burden of infectious diseases on public health systems, ${ }^{22}$ and the most successful method of morbidity reduction in history, after access to clean water. ${ }^{23}$ As such, immunizations represent an essential ingredient for the survival, strengthening and stability of political systems and economies worldwide. ${ }^{24}$ Unsurprisingly, the Lancet identified them as the single most important intervention for controlling the spread of influenza pandemics. ${ }^{25}$ Their efficacy and efficiency has earned vaccines the sobriquet of formidable 'tools' for advancing public

\footnotetext{
${ }^{15}$ Morten Broberg, 'A Critical Appraisal of the World Health Organization's International Health Regulations (2005) in Times of Pandemic: It is Time for Revision' (2020) 11 European Journal of Risk Regulation 202; Lawrence Gostin and Rebecca Katz, 'The International Health Regulations: The Governing Framework for Global Health Security' (2016) 94 The Millibank Quarterly 264; Lawrence Gostin, Mary De Bartolo and Eric Friedman, 'The International Health Regulations 10 Years on: The Governing Framework of Global Health Security' (2015) 386 The Lancet 2222.

${ }^{16}$ Broberg, 'A Critical Appraisal of the World Health Organization's International Health Regulations (2005)' (n 12).

${ }^{17}$ Health Threats Decision (n 8) art 5-14; see also Andrea Renda and Rosa Castro, 'Towards Stronger EU Governance of Health Threats after the COVID-19 Pandemic' (2020) 11 European Journal of Risk Regulation 273.

18 WHO, 'A Safer Future: Global Public Health Security in the $21^{\text {st }}$ Century', The World Health Report 2007.

${ }^{19}$ D Fidler, 'A Pathology of Public Health Securitism: Approaching Pandemics as a Security Threat', in A Cooper (ed.), Governing Global Health: Challenge, Response, Innovation (Routledge 2016); Andrew Dobson, Keiza Barker and Sarah Taylor, Biosecurity: The Socio-Politics of Invasive Species and Infectious Diseases (Routledge 2013).

${ }^{20}$ Hylke Dijkstra and Anniek de Ruijter, 'The Health-Security Nexus and the European Union: Towards a Research Agenda' (2017) 8 European Journal of Risk Regulation 613.

21 ibid 614; Renda and Castro (n 17).

${ }^{22}$ Shabaana Khader and Jay Kolls, 'Vaccines for Infectious Diseases: Are we There Yet?' (2014) 28 Current Opinion in Immunology ix.

${ }^{23}$ F Andre and others, 'Vaccination Greatly Reduces Disease, Disability, Death and Inequity Worldwide' (2008) 86 Bulletin of the World Health Organization 140; Mark Doherty and others, 'Vaccine Impact: Benefits for Human Health' (2016) 34 Vaccine 6707.

${ }^{24}$ David Bloom, David Canning and Mark Weston, 'The Value of Vaccination' (2005) 6 World Economics-Henley on Thames 15.

${ }^{25}$ The Lancet (n 1).
} 
health and other public policy goals, particularly given the unique combined individual and collective protection they provide against infectious diseases through 'herd immunity'. ${ }^{26}$

Now, if preparedness is to be understood as a 'tool box' to confront emerging health threats, one could legitimately expect vaccines (from discovery to deployment) to feature prominently in the governance of public health preparedness. Yet, therapeutic countermeasures only feature in Art. 5 of the Health Threats Decision for the purpose of joint procurement and stockpiling ${ }^{27}$ - both of which, by definition, require the vaccine or therapy to be already available. The development of such countermeasures is not strategically embedded in the framework, and this constitutes a major flaw made blatantly obvious by the current COVID-19 pandemic. The core of the problem is the absence of vaccine R\&D and dedicated regulatory approval pathways as integral elements of public health preparedness. At the international level, vaccines are similarly absent from the IHR 2005 . This has attracted criticism that the regulations need a greater 'One-Health' focus, ${ }^{28}$ entailing emphasis on facilitating vaccine R\&D as well as integration of the IHR 2005 with separate dedicated programs such as the WHO Pandemic Influenzas Preparedness Framework, and the Global Vaccine Action Plans $^{29}$ - which, however, tend to be more focused on access than R\&D. ${ }^{30}$

Both the IHR 2005 and the EU Health Threats Decision share fundamental features, arguably stemming from the rise of the health-security nexus. In broad terms, they are reactive in nature and focused on the ability to detect, assess and respond to events. ${ }^{31}$ At its core, the issue seems to be that both frameworks have internalised a neat distinction between what constitutes precautionary or preventative action from what is specifically preparatory action, focused on the aftermath of an unstoppable event with the aim of minimising disruption. ${ }^{32}$ In this sense, the confinement of therapeutic countermeasures to stockpiling and procurement provisions obeys an implementation pattern whereby preparedness is constructed through 'past and current actualisation', 33 which is vulnerable to the unexpected and unanticipated. ${ }^{34}$ The COVID-19 pandemic makes clear that this compartmentalised logic creates distinctions that are, in the realm of public health, both artificial and harmful: being prepared for emerging PEDs cannot be divorced from the research and development of immunization tools.

\footnotetext{
${ }^{26}$ M Sujitha, 'Vaccines as the Medical Weapon for a Promised Healthy Generation' (2020) 6 IOSR Journal of Biotechnology and Biochemistry 1; Peter Hoetz, "Vaccine Diplomacy": Historical Perspectives and Future Directions' (2014) 8(6) PLoS Neglected Tropical Diseases e2808; Robert Breiman, 'Vaccines as Tools for Advancing more that Public Health: Perspectives of a Former Director of the National Vaccine Program Office' (2001) 32 Clinical Infectious Diseases 283.

27 Health Threats Decision (n 8) art 5 on Joint procurement of medical countermeasures.

${ }^{28}$ Gostin and Katz (n 15) 286.

${ }^{29}$ Gostin, De Bartolo and Friedman (n 15).

30 Line Matthiessen and others, 'European Strategy for Vaccine Development Against Infectious Diseases' (2016) 35 Vaccine A20; Odile Leroy and others, 'Roadmap for the Establishment of a European Vaccine R\&D Infrastructure' (2014) 32 Vaccine 7021.

${ }^{31}$ Rebecca Katz and Erin Sorrell, 'Preparedness and Resilience in Public Health Emergencies', in Simon Rushton and Jeremy Youde (eds), Routledge Handbok of Global Health Security (Routledge 2014) 202.

32 Ben Anderson, 'Preemption, Precaution, Preparedness: Anticipatory Action and Future Geographies' (2010) 34 Progress in Human Geography 777.

33 Jose Canada, 'Hybrid Threats and Preparedness Strategies: The Reconceptualization of Biological Threats and Boundaries in Global Health Emergencies' (2019) 24 Sociological Research Online 93, 96.

${ }^{34}$ Anderson (n 32) 782.
} 


\section{Researching and approving vaccines: regulatory gaps and past lessons}

Having a new vaccine approved for marketing is a complex and bumpy process that can take up to fifteen years. This is due to a number of factors including the scale and diversity of the target population of vaccines, which mandates reliance on very large safety and efficacy datasets. ${ }^{35}$ In the EU, no dedicated regulatory pathway exists for the development and approval of vaccines, which undergo the same regime as other pharmaceutical products established under Directive 2001/83/EC. ${ }^{36}$ As recently highlighted by a study in the New England Journal of Medicine, ${ }^{37}$ a significant issue during a pandemic is that mass production typically takes place after a marketing approval has been obtained, whereas the public health emergency triggered by a PED outbreak demands prompter availability, requiring large scale manufacturing to begin while clinical development is still ongoing. This is however incompatible with the economic model that underpins vaccine $R \& D$, which is dependent on investment from a private sector reluctant to risk significant production investments ahead of approvals. ${ }^{38}$

In the wake of COVID-19, the European Medicines Agency (EMA), has adopted a number of initiatives to streamline the administrative burden that accompanies manufacturers during the preapproval process, ${ }^{39}$ and to coordinate with foreign authorities for the purpose of accelerating mutual recognition of clinical trial data internationally. ${ }^{40}$ The European requirements of product safety and efficacy laid out in the Medicines Directive remain substantially unchanged. ${ }^{41}$ This can be contrasted with the US regulatory regime set out under the Public Readiness and Emergency Preparedness Act (PREP) Act, ${ }^{42}$ allowing the Food and Drug Administration (FDA) to issue immunities from liability for industry involved in the manufacture, testing, development and distribution of effective pandemic countermeasures. ${ }^{43}$ The US's approach to the development of pandemic countermeasures accepts the higher degrees of risk attached to therapeutic countermeasures as an acceptable trade-off for speedier availability. At an international level, this is mirrored by the WHO Emergency Use Assessment and Licensing Procedure (EUAL), which is described as a "special procedure for vaccines in the case of a public health emergency when the community may be more willing to tolerate less certainty about the efficacy and safety of products." ${ }^{44}$ The EUAL procedure can function as a blueprint for WHO member states intending to fast-track the approval of new vaccines in the course of PED outbreaks. ${ }^{45}$

\footnotetext{
${ }^{35}$ Marco Rizzi, 'The Road to a Vaccine for COVID-19: Regulatory and Policy Infrastructure, Incentives and Obstacles' (2020) 4 European Pharmaceutical Law Review 98.

${ }^{36}$ Directive 2001/83/EC of the European Parliament and of the Council on the Community code relating medicinal products for human use (Medicines Directive) [2001] OJ L 311.

${ }^{37}$ Lurie and others ( $\left.n 7\right)$.

38 Ibid.

39 EMA, 'EMA initiatives for acceleration of development support and evaluation procedures for COVID-19 treatments and vaccines' (4 May 2020) EMA/213341/2020.

40 EMA, 'International regulators align positions on phase 3 COVID-19 vaccine trials' (9 July 2020) EMA/367602/2020.

${ }^{41}$ Duncan Fairgrieve and others, 'Products in a Pandemic: Liability for Medical Products and the Fight against COVID19' (2020) European Journal of Risk Regulation (online first); Rizzi, 'The Road to a Vaccine for COVID-19' (n 35).

42 (2005) 42 USC $\$ 247 d-6 d$.

43 Fairgrieve and others (n 41) 35-36.

${ }^{44}$ WHO, 'Emergency Use Assessment and Licensing Procedure (EUAL) for candidate vaccines for use in the context of a public health emergency' (7 July 2015) <https://www.who.int/medicines/news/EUALvaccines_7July2015_MS_(Updated_notes-disclaimers_21Aug2018).pdf?ua=1> accessed 3 October 2020.

${ }^{45}$ Marco Rizzi, 'Vaccines for Pandemic and Epidemic Diseases: Towards Defining the Space of EU Public Health between Security Policy and a Transnational Market' (2017) 8 European Journal of Risk Regulation 686, 694.
} 
In the absence of a broader approach, the EU regulatory framework features two special procedures to face potential influenza pandemics: the mock-up procedure (which permits vaccine development in advance of pandemics based on information from virus strains that could cause PEDs) and the emergency procedure (which streamlines approval once a pandemic has been declared) - leading up to conditional approvals, the one concession made by EU regulations to the cost-benefit calculus of immediate availability outweighing the risk of less comprehensive data. The procedures remain however largely limited to usage in the face of influenza pandemics. ${ }^{46}$

Alongside regulatory limitations, the development of new vaccines suffers from a stale incentive structure. The two feed off each other, regulatory requirements having long been identified as a structural obstacle to innovation in the space of emerging diseases. ${ }^{47}$ In synthesis, the issue is that, where private industry plays a central role, the development of new vaccines is inextricably tied to three factors: need, availability and cost. ${ }^{48}$ Despite the efforts of the WHO in identifying infectious diseases with the potential to cause future outbreaks, market incentives have consistently failed to support R\&D. ${ }^{49}$ Current regulatory pathways have also been singled out as difficult to adapt to the PED context for a number of reasons, including reliance on vaccine developers to invest significant resources with no guarantees or risk sharing mechanisms. ${ }^{50}$ Heavy reliance on the market in such a critical aspect of public health protection is at the root of increasing societal dependence on 'philantrocapitalism', ${ }^{51}$ or other hybrid forms of public-private partnerships such as the Coalition for Epidemic Preparedness Innovations (CEPI). ${ }^{52}$ To grasp what the combined effect of current market incentives and regulatory pathways means for vaccine development, it is useful to look at recent PED outbreaks.

In March 2009, the A-H1N1 influenza pandemic (the 'Swine Flu') started in Mexico and quickly spread globally. Within 32 days of the WHO declaring a Public Health Emergency of International Concern (PHEIC), the first vaccine candidate was developed, ${ }^{53}$ within five months the first vaccine was licensed, and by the end of the year millions of doses had been produced. ${ }^{54}$ The EMA had been developing pandemic influenza vaccines since 2003 and set up the mock-up procedure in 2004. This led to the pre-approval of four mock-up vaccines ahead of the pandemic, which were evaluated on a rolling basis as the pandemic unfolded. ${ }^{55}$ The subsequent production and distribution was not

46 ibid 695; Sumimasa Nagai, 'Flexible and Expedited Regulatory Review Processes for Innovative Medicines and Regenerative Medical Products in the US, the EU, and Japan' (2019) 20 International Journal of Molecular Sciences 3801. Emergency procedures also includes seriously debilitating or life-threatening diseases.

47 Jacqueline Keith and others, 'Delivering the Promise of the Decade of Vaccines: Opportunities and Challenges in the Development of High Quality New Vaccines' (2012) 31 Vaccine B184.

48 Joel Maslow, 'Challenges and solutions in the development of vaccines against emerging and neglected infectious diseases' (2019) 15 Human Vaccines \& Immunotherapeutics 2230.

49 Dimitrios Gouglas and Kevin Marsh, 'Prioritizing Investments in New Vaccines Against Epidemic Infectious Diseases: A Multi-criteria Decision Analysis' (2019) 26 Journal of Multi-Criteria Decision Analysis 153.

50 John-Arne Røttingen and others, 'New Vaccines Against Epidemic Infectious Diseases' (2017) 376 The New England Journal of Medicine 610.

51 Yuyang Mei, 'Neoliberal Optimism: Applying Market Techniques to Global Health' (2017) 36 Medical Anthropology 381.

52 Dimitrios Gouglas and others, 'Estimating the Cost of Vaccine Development Against Epidemic Infectious Diseases: a Cost Minimisation Study' (2018) 6 The Lancet Global Health e1386.

53 Harvey Fineberg, 'Pandemic Preparedness and Response - Lessons from the H1N1 Influenza of 2009' (2914) 370 The New England Journal of Medicine 1335.

54 Jeffrey Partridge and Marie Paul Kieny, 'Global production of seasonal and pandemic (H1N1) influenza vaccines in 2009-2010 and comparison with previous estimates and global action plan targets' (2010) 28 Vaccine 4709.

${ }^{55}$ Germaine Hanquet and others, 'Lessons Learnt from Pandemic A(H1N1) 2009 Influenza Vaccination. Highlights of a European Workshop in Brussels (22 March 2010)' (2010) 29 Vaccine 370. 
without criticism, ${ }^{56}$ but from the point of view of the prompt availability of a vaccine, the Swine Flu was a remarkable success.

The Ebola outbreak, which started in West Africa in 2014, tells a different story. By 2009, as many as seven vaccines had undergone pre-clinical testing with encouraging results, yet only one reached Phase I of clinical trials and was subsequently abandoned for being considered unlikely to provide sufficient return on investment. ${ }^{57}$ Even after the outbreak was declared a PHEIC in August 2014, development was slow to start due to lack of infrastructure and funding. ${ }^{58}$ Ebola prompted funding agencies, academic institutions and segments of the industry to accelerate vaccine development more than ever before. ${ }^{59}$ Yet, the underlying bias against investing in a disease that occurs only sporadically and typically in rural areas not affecting affluent countries, ${ }^{60}$ caused significant initial R\&D delays, with a vaccine reaching conditional approval in November 2019. Ebola also revealed the absence of sufficient incentives for coordinating R\&D activities, highlighting the prevalence of market-based competition even in the context of a PHEIC. ${ }^{61}$ The fate of vaccine R\&D during the SARS and MERS outbreaks of 2002 and 2012 corroborates these observations. ${ }^{62}$ Somewhat ironically, research into vaccines for these coronaviruses was abandoned on financial grounds, depriving scientists of a potential head-start in the search for a SARS-CoV2 vaccine. ${ }^{63}$

\section{Shifting paradigm in the regulation of PEDs vaccines R\&D}

These concluding observations are based on the premise that European public health authorities need to include proactive R\&D of vaccines for potential PEDs as an integral part of their preparedness strategies. To this end, a future European Health Union should embrace a bold paradigm shift in vaccine R\&D as the COVID-19 pandemic is putting the current combination of regulatory infrastructure and economic incentives under unprecedented pressure, laying bare weaknesses that previous outbreaks had already suggested. The disastrous damage to both the economies and social fabrics of EU Member States stands as a stark admonishment of the temptation to return to some semblance of 'business as usual'. The following suggestions are certainly underdeveloped, but they are meant to push the debate in a direction that may ultimately better equip a European Health Union to face the unexpected in the future.

Vaccine R\&D cannot depend on private investment as much as it currently does, and needs significant and structured public funding. While this may not have been a realistic prospect until but a few months ago, the recent adoption of the Next Generation EU Plan with a dedicated Health

\footnotetext{
${ }^{56}$ Francisco Bombillar, 'The case of pandemic flu vaccines: Some lessons learned' (2010) 1 European Journal of Risk Regulation 427.

57 Stanley Plotkin, Adel Mahmoud and Jeremy Farrar, 'Establishing a Global Vaccine-Development Fund' (2015) 373 The New England Journal of Medicine 297.

${ }^{58}$ Ana Maria Henao-Restrepo and others, 'On a Path to Accelerate Access to Ebola Vaccines: The WHO's Research and Development Efforts During the 2014-2016 Ebola Epidemic in West Africa' (2016) 17 Current Opinion in Virology 138.

${ }^{59}$ Adam Gristwood, 'Fresh Approaches to Vaccine Development: New Financial and Economic Models Are Required to Bring More Vaccines Against a Wider Range of Diseases to the Market' (2018) 19 EMBO Reports e46675.

60 Seth Berkley, 'Share the Risks of Ebola Vaccine Development: Ebola Vaccines Have Little in the Way of Commercial Markets, so the Risks Should Be Shared Between Governments and Industry' (2015) 519 Nature 263.

61 Renda and Castro (n 17) 276.

62 Rachel Roper and Kristina Rehm, 'SARS Vaccines: Where Are We?' (2009) 8 Expert Review of Vaccines 887; Stanley Perlman and Rahul Vijay, 'Middle East Respiratory Syndrome Vaccines' (2016) 47 International Journal of Infectious Diseases 23; Eva Riedmann, 'MERS Vaccine Is Technically Feasible, but Is It Commercially Feasible?' (2014) 10 Human Vaccines \& Immunotherapeutics 1429.

${ }^{63}$ Liam Mannix, 'We should have had vaccine already: Australian expert who cracked virus code' The Sydney Morning Herald (Sydney, 28 March 2020) <https://www.smh.com.au/national/we-should-have-had-vaccine-alreadyaustralian-expert-who-cracked-virus-code-20200327-p54elr.html> accessed 3 October 2020.
} 
Programme demonstrates that old preconceptions are being challenged. ${ }^{64}$ Research into vaccines for potential PEDs should become a strategic pillar of the European 'entrepreneurial state'. ${ }^{65}$ The 'mock-up' procedure, currently limited to influenza viruses, can form the blueprint of future regulatory developments to provide concrete means of R\&D based on wide ranging research into emerging pathogens that could cause PED outbreaks - thus divorcing R\&D from necessary returns on investment. In the occurrence of a PED, as is the case with current conditional approvals, safety and efficacy data would have to be reviewed on a rolling basis to assess potentially competing mockup vaccines, and this should be complemented by mandatory cooperative provisions for data sharing, with cooperation taking precedence over market-based competition. Finally, given the inevitable trade-offs between risk and availability inherent to relying on mock-up vaccines, it would be appropriate for the Health Union to set up a public no-fault compensation scheme for potential injuries linked to such vaccinations.

These are preliminary proposals, requiring much refinement and fleshing out. They also, admittedly, are costly and challenge deeply rooted legal and economic structures and beliefs. It also bears noting that at the time of writing, things are moving and changing at a breakneck speed. For example, in late July the Commission announced a substantial contribution to the efforts of CEPI. ${ }^{66}$ It is also calculated that up to September 2020 the EU invested $€ 459$ million in projects targeting the pandemic (including, but not limited to, vaccine R\&D). ${ }^{67}$ These initiatives are certainly positive and are a further indication of an ongoing shift. But the underpinning logic is still very much anchored to ex post response to emergency as opposed to strategic ex ante preparedness planning. These observations are not meant to trivialise the remarkable efforts of EU institutions in responding to the current unprecedented crisis. Rather, they are aimed at restructuring future governance models, priorities and design in a world that, as we now painfully know, can be brought to a halt by an emerging PED. It will be for the future Health Union to rise to the challenge of rethinking vaccine development as integral to public health preparedness, and take the lead in governing the legal, political and economic implications of this complex but necessary shift.

\footnotetext{
${ }^{64}$ See European Union, 'The pillars of Next Generation EU' (European Commission, 10 June 2020) <https://ec.europa.eu/info/live-work-travel-eu/health/coronavirus-response/recovery-plan-europe/pillars-nextgeneration-eu_en> accessed 3 October 2020. The Health Programme features under Pillar 3: Learning the lessons from the crisis.

${ }^{65}$ Mariana Mazzucato, The Entrepreneurial State - Debunking Public vs Private Sector Myths (Penguin 2018).

${ }^{66}$ European Commission, 'Coronavirus: EU supports vaccine research with additional $€ 100$ million' (NEWS, 22 July 2020) <https://ec.europa.eu/info/news/coronavirus-eu-supports-vaccine-research-additional-eu100-million-2020jul-22_en> accessed 3 October 2020.

${ }^{67}$ European Union, 'EU research and innovation in action against the coronavirus: funding, results and impact' (European Commission, September 2020) <https://ec.europa.eu/info/sites/info/files/research_and_innovation/research_by_area/documents/ec_rtd_corona virus-research-projects-overview.pdf> accessed 3 October 2020.
} 